\title{
BMJ Open Effectiveness of integrated chronic care interventions for older people with different frailty levels: a systematic review protocol
}

\author{
Nimra Khan (1) , David Hewson (1) , Gurch Randhawa (1)
}

To cite: Khan N, Hewson D, Randhawa G. Effectiveness of integrated chronic care interventions for older people with different frailty levels: a systematic review protocol. BMJ Open 2020;10:e038437. doi:10.1136/ bmjopen-2020-038437

- Prepublication history for this paper is available online. To view these files, please visit the journal online (http://dx.doi. org/10.1136/bmjopen-2020038437).

Received 10 March 2020 Revised 14 July 2020 Accepted 31 July 2020

\section{Check for updates}

(C) Author(s) (or their employer(s)) 2020. Re-use permitted under CC BY-NC. No commercial re-use. See rights and permissions. Published by BMJ.

Institute for Health Research, University of Bedfordshire, Luton, UK

Correspondence to Dr Gurch Randhawa gurch.randhawa@beds.ac.uk

\section{ABSTRACT}

Introduction Frailty poses a huge burden to individuals, their families and to healthcare systems. Several interventions have been evaluated for the improvement of outcomes for older people with frailty, including integrated care interventions. Reviews synthesising evidence on the effectiveness of integrated care for older people with frailty have treated them as a single population, without considering the heterogeneity between different frailty levels such as non-frail, mild frailty, moderate frailty and severe frailty. Findings from these studies have shown inconsistent results on the various outcomes assessed. People with different frailty status have different care needs, which should be addressed accordingly. The aim of this study is to synthesise evidence on the effectiveness of integrated care interventions on older people with different frailty status who are community dwelling or living in retirement housing or residential setting but not in care homes or in nursing homes.

Methods and analysis This is a protocol for a systematic review assessing the effectiveness of integrated chronic care interventions on older people with different frailty status. A literature search will be conducted on the databases Cochrane Central Register of Controlled Trials, PubMed, Embase, Web of Science, Cumulative Index to Nursing and Allied Health Literature, and clinical trial registers. Two authors will independently conduct search and screening for eligible studies. Full-text screening will be used to include only studies that fulfil the inclusion criteria. Data extraction will be done on a data extraction form and methodological quality of studies will be assessed using the Effective Practice and Organisation of Care risk of bias tool. The interventions will be described following Wagner's Chronic Care Model.

Ethics and dissemination Ethical approval for this study was obtained from the Institute for Health Research Ethics Committee of the University of Bedfordshire (IHREC934). The results of the review will be disseminated through a peer-reviewed journal article, conferences and also with local provider and user stakeholders.

\section{PROSPERO registration number CRD42020166908.}

\section{BACKGROUND}

Frailty is a state characterised by decreased physiological reserves due to an age-related accumulation of deficits, which makes an individual
Strengths and limitations of this study

- To our best knowledge this is the first systematic review which will stratify older people based on their frailty status.

- The study will map integrated care interventions on the chronic care model.

- The protocol is based on the Preferred Reporting Items for Systematic Reviews and Meta-Analysis Protocol guidelines.

- Essential steps such as screening of studies, data extraction and quality assessment will be done in duplicate.

- Databases in languages other than English will not be searched or included, which may cause language bias.

vulnerable to minor stressors. ${ }^{1-3}$ It is associated with adverse outcomes such as falls, fractures, emergency hospital admissions, institutionalisation and mortality. Frailty is more common among women than men, higher among older age groups, higher among some ethnic groups, as well as in people from low socioeconomic background, having less education and higher poverty. ${ }^{45}$

There is some evidence that frailty can be reversed if identified at an earlier stage of the process. ${ }^{6}{ }^{7}$ Several interventions that can be broadly categorised into single component, multi-domain and integrated care have been evaluated to improve outcomes for older people with frailty. Single component interventions are those that include only one component, such as exercise. A scoping review by Puts $e t a l^{5}$ and systematic reviews by Apostolo $e a^{8} l^{8}$ and Daniels et al found that interventions with exercise were effective in preventing or reducing frailty in frail and pre-frail individuals. However, these studies did not provide pooled estimates or effect sizes. Some recently conducted randomised controlled trials (RCTs) ${ }^{10-12}$ have also reported exercise interventions to be effective in reducing 
frailty. However, evidence on the effect of other single component interventions such as nutrition is inconsistent. ${ }^{1314}$ Multidomain interventions refer to those interventions that have two or more components. The most commonly reported multi-domain interventions are based on a combination of nutrition and exercise, with systematic reviews summarising evidence on these combinations reporting them to be effective. ${ }^{51516}$ A number of RCTs ${ }^{17-19}$ that assessed multi-domain interventions, in which physical activity and nutrition have been complemented by cognitive training, have shown a reduced risk of developing frailty as well as improvement in frailty status among older people and increases in functional status.

There are a growing number of systematic reviews that have evaluated integrated care interventions for frailty. ${ }^{20-24}$ Integrated care has been defined as an organisational approach of coordinating continuous care based on a patient's needs and viewing the patient in a holistic manner. ${ }^{25}$ All of the systematic reviews on integrated care have considered older people with different levels of frailty as a single population and did not distinguish by frailty status. However, there is evidence that older people with different frailty status have different care needs, require different types of interventions and respond differently to interventions. ${ }^{26}$ Therefore, treating them as a single population may be one reason for the heterogeneity in the outcomes reported in systematic reviews of integrated care interventions.

The aim of this systematic review is to synthesise evidence on the effectiveness of integrated care interventions on older people with different frailty status, including those living in both the community and in residential care settings. To this end, the proposed systematic review will answer the following question:

Are integrated care interventions effective in preventing or managing frailty among older people with different frailty levels as compared to the usual care?

\section{METHODS AND ANALYSIS Eligibility criteria}

Studies will be included in the review if they meet the following inclusion criteria:

\section{Participants}

People aged 65 years old and above classified as frail using a valid frailty assessment instrument such as accumulation of deficits model or the frailty phenotype model as described by the trial authors. Participants must be classified according to their frailty level by the assessment tool used.

\section{Interventions}

Integrated chronic care models are introduced to overcome the current fragmentation in healthcare system. It aims to move away from disease-oriented approach to patientcentred care by offering services based on the needs, preferences and choices of individuals. ${ }^{27}$ Since older people can be stratified based on their frailty levels, those identified as robust or pre-frail include older adults without complex care needs; whereas, those who are moderately frail have higher level of frailty and are at increased risk of developing complex care needs. The older adults who are severely frail have complex care needs. ${ }^{28}{ }^{29}$ Therefore, we will include studies in which a population health management model has been used, for example the Kaiser Permanente model, to stratify community-dwelling older people into risk profiles based on their levels of frailty, with care and support offered using multidisciplinary teams with the intensity determined by the individual risk profile and care needs. For example, those identified as robust or having mild frailty could be offered self-management support, healthy lifestyle advice and participation in physical activity programmes. Those with moderate frailty could be provided with case management support from various healthcare professionals such as general practitioners and district nurses, with social care workers as case managers and coordinating follow-up. Older people who are severely frail or have high complexity could receive intensive case management. Several frameworks have been developed to improve the understanding of the key elements of a successful integrated care programme. One of the most influential among them is the chronic care model (CCM), which is an evidence-based conceptual framework that provides guidance on the organisation of healthcare for people with chronic conditions to improve their outcomes. ${ }^{30} 31$ The CCM, which has been acknowledged by the WHO, includes six elements: (a) providing support to patients for self-management; (b) decision-making support to providers; (c) case management; (d) establishing a clinical information system; (e) community resources for healthy living and (f) leadership within the health system. ${ }^{32}$

The interventions in included studies will be described using the taxonomy of the CCM, whereby elements of each intervention will be mapped on the elements of CCM, as described by Wagner, ${ }^{30}$ since frailty shares many features with a chronic condition. For example, frailty is a dynamic syndrome. There is some evidence that suggests that frailty is reversible if identified and interventions provided at an earlier stage of the process. However, less is known about reversibility of frailty among those who are severely frail or have complex care need. ${ }^{67}$ It can be prevented and better managed through the action of an interdisciplinary approach that proactively organises and coordinates care to prevent the associated adverse outcomes. ${ }^{32}$ The CCM provides a comprehensive framework to manage long-term conditions such as frailty by covering all essential elements of integrated care. ${ }^{33}$ Therefore, by mapping the interventions on the six elements of the CCM, we will be able to examine whether interventions contain the essential components of integrated care services and also their association with outcomes.

\section{Comparator(s)}

Interventions must be compared with usual care.

\section{Outcomes}

\section{Primary outcomes}

- Falls: the WHO has defined falls as an involuntary event bringing the body to the ground or other surfaces. ${ }^{34}$ 
- Emergency hospital admission is when a person is admitted to hospital urgently and unexpectedly, that is, the admission is unplanned. ${ }^{35}$

- Quality of life (QoL) is a complex concept and its precise definition is debated. The WHO has described QoL as 'an individual's perceptions of their position in life, in the context of the culture and value systems in which they live, and in relation to their goals, expectations, standards and concerns' ${ }^{36}$ Due to diverse definitions of QoL, we will include studies that have used valid instruments such as the Older People's QoL Questionnaire, ${ }^{37}$ the Short Form-36 health-related QoL tool, ${ }^{38}$ the WHO QoL Assessment Instrument ${ }^{39}$ to measure and report on outcomes such as 'quality of life', 'well-being' or 'life satisfaction'.

- Institutionalisation is defined as when individuals who are no longer capable of living independently in their own home, are provided with accommodation and care support in institutional settings. ${ }^{40}$

- Mortality.

- Transitioning in frailty status such as shift from robust to pre-frailty, from pre-frailty to frailty or vice versa. ${ }^{2641}$ We justify inclusion of these outcomes based on existing studies, which have stated that frailty is associated with adverse outcomes such as falls, emergency hospital admission, poor QoL, institutionalisation and mortality. ${ }^{3} 1415242642-51$ Furthermore, studies have shown that frailty is a condition, which can transition from better to worst or vice versa. ${ }^{52}$

\section{Secondary outcomes}

- Physical disability measured by screening for ability to perform self-care tasks such as activities of daily living and tasks of household management like instrumental $\mathrm{ADL}$ or any other valid measurement as stated by the trial authors. It has been included because frailty is identified as a risk factor for physical disability. ${ }^{42}$

- Carers' burden measured using valid instruments such as the Zarit Burden Interview ${ }^{53}$ will be included.

- Healthcare utilisation and cost effectiveness ${ }^{24}$ as stated by trial authors.

\section{Studies}

Study designs considered will be quantitative empirical studies with a control group including RCTs of any design such as those with individual or cluster randomisation and quasi-experimental designs.

\section{Search strategy \\ Electronic databases \\ - Medline. \\ - Embase. \\ - Cumulative Index to Nursing and Allied Health Liter- ature (CINAHL). \\ - Web of Science. \\ Clinical trial registers \\ - Cochrane Central Register of Controlled Trials. \\ - ClinicalTrials.gov.}

WHO International Clinical Trials Registry Platform.

Other sources

- Reference lists of the included studies.

- Systematic reviews on similar topics and their reference lists.

\section{Keyword searches}

The search strategy will use free words as well as Medical Subject Headings for Medline and CINAHL. Two authors (NK and DH) will independently carry out the search during June-July 2020. An example of the search strategy for Medline is shown in online supplemental material .

\section{Inclusion and exclusion criteria}

There will no time limitation. Articles will be limited to English, but no geographical locations will be specified. Furthermore, studies having older people with different levels of frailty who are either community dwelling or living in retirement housing or residential setting but not care homes or nursing homes will be included.

Studies evaluating interventions other than integrated care will be excluded. Qualitative studies will be excluded. Studies that have used frailty assessment tools that do not distinguish the severity of frailty will be excluded.

\section{Data extraction}

Studies identified will be imported into reference management software EndNote V.X9.3 (Clarivate, Philadelphia, Pennsylvania, USA) for deduplication and filtering. Two reviewers (NK and $\mathrm{DH})$ will independently screen the titles and abstracts of the studies fulfilling eligibility criteria. The articles will be categorised into three groups: relevant, irrelevant and unsure. Articles categorised as irrelevant by both reviewers will be rejected. Each reviewer will assess the full text of the remaining articles and make a list of articles to be included. Any disagreements will be resolved by involving a third reviewer (GR). Full-text versions of the remaining articles will be assessed by using the Cochrane Effective Practice and Organisation of Care Review Group (EPOC) standard data collection checklist, which will be adapted for data extraction. Data will be extracted on study design, participant characteristics (age, gender and level of education), intervention characteristics, location of care (community or residential setting), country, primary and secondary outcomes, source of funding and others. Furthermore, information on intervention fidelity such as adherence, frequency, duration, coverage and other elements mentioned by trial authors will be extracted. Two review authors (NK and $\mathrm{DH}$ ) will independently extract the study characteristics from the primary studies included in the review using a customised Microsoft Excel table, with article selection based on Population, Intervention, Comparison and Outcomes elements. Two review authors (NK and $\mathrm{DH}$ ) will extract outcomes data from the included studies, with any disagreements on the outcomes data decided by a third reviewer (GR), in accordance with the Cochrane Handbook for Systematic Reviews of Interventions. ${ }^{54}$ 


\section{Risk of bias assessment}

Risk of bias will be evaluated using the EPOC risk of bias tool, ${ }^{55}$ which is suitable for this review because the method also includes non-randomised trials. This tool has nine criteria, including random sequence generation, allocation concealment, baseline characteristics, outcome measures at baseline, incomplete outcome data, knowledge of allocated intervention, protection against contamination, selective reporting of outcomes and other risks of bias. For each of the nine domains, the procedures undertaken by individual studies will be described, including verbatim quotes. Two review authors (NK and $\mathrm{DH}$ ) will independently assess the risk of bias and categorise the studies as having low risk, high risk or unclear risk of bias. Any disagreements will be resolved by involving a third reviewer (GR). Graphic representations of potential bias within and across studies will be computed using Review Manager V.5.4 (The Nordic Cochrane Centre, The Cochrane Collaboration, Copenhagen, Denmark). Each item in the risk of bias assessment will be considered independently, without an attempt to collate and assign an overall score.

\section{Data synthesis}

Quantitative data will, where possible, be pooled in statistical meta-analysis. All results will be subject to double data entry. Effect sizes expressed as OR or relative risk (for categorical data) and weighted mean differences (for continuous data) and their $95 \%$ CIs will be calculated for analysis. Heterogeneity will be assessed statistically using the standard $\chi^{2}$ and also explored using subgroup analyses based on the different quantitative study designs included in this review. Furthermore, we will use L'Abbé plot to explore heterogeneity and identify outlying trials in a meta-analysis. ${ }^{56}$

Quality of evidence will be assessed using Grades of Recommendation, Assessment, Development and Evaluation. ${ }^{57}$ Sensitivity analysis will be performed by removing studies with higher risk of bias. When statistical pooling is not possible, the findings will be presented in narrative form including tables and figures to aid in data presentation where appropriate. These results will be combined to arrive at a conclusion from the research. After performing data synthesis, the final report will be prepared following the Preferred Reporting Items for Systematic Reviews and Meta-Analyses guidelines. Furthermore, in case of any deviations from the protocol the authors will mention them in the final published report and update in the PROSPERO record.

\section{DISCUSSION}

Despite a plethora of systematic reviews conducted on synthesising evidence on the effectiveness of integrated care interventions for older people, all such reviews have treated older people with different levels of frailty as a single population. This could be one reason for the heterogeneity in findings from the existing reviews. This review will fill this gap by synthesising evidence on the effectiveness of integrated care interventions for older people with different frailty levels.

\section{Dissemination}

The findings of this review will be shared through a peerreviewed journal article, conferences, and with local commissioners and stakeholders involved in providing integrated care services for the older population.

\section{Ethical issues}

This is a systematic review that will only use data from existing studies, all of which will have obtained ethical approval. As such, there are no ethical considerations for the project, however, data collected from the studies included in the review will be treated with due consideration.

Contributors NK conceived the idea, planned and wrote the first draft of the study protocol. DH developed the search strategy. The first draft was reviewed by DH and GR. DH and GR provided critical insights and drafting comments. All authors have approved and contributed to the final written manuscript. The guarantor of the review will be $\mathrm{DH}$.

Funding Luton Clinical Commissioning Group and University of Bedfordshire supported this study. The funding body had no role in developing the protocol. NK is recipient of a PhD scholarship from the Luton Clinical Commissioning Group and the Institute for Health Research of the University of Bedfordshire.

Competing interests None declared.

Patient and public involvement Patients and/or the public were involved in the design, or conduct, or reporting, or dissemination plans of this research. Refer to the Methods section for further details.

Patient consent for publication Not required.

Provenance and peer review Not commissioned; externally peer reviewed.

Open access This is an open access article distributed in accordance with the Creative Commons Attribution Non Commercial (CC BY-NC 4.0) license, which permits others to distribute, remix, adapt, build upon this work non-commercially, and license their derivative works on different terms, provided the original work is properly cited, appropriate credit is given, any changes made indicated, and the use is non-commercial. See: http://creativecommons.org/licenses/by-nc/4.0/.

\section{ORCID iDs}

Nimra Khan http://orcid.org/0000-0002-6944-4879

David Hewson http://orcid.org/0000-0002-7656-4000

Gurch Randhawa http://orcid.org/0000-0002-2289-5859

\section{REFERENCES}

1 Xue Q-L. The frailty syndrome: definition and natural history. Clin Geriatr Med 2011;27:1-15.

2 Cesari M, Prince M, Thiyagarajan JA, et al. Frailty: an emerging public health priority. J Am Med Dir Assoc 2016;17:188-92.

3 Clegg A, Young J, lliffe S, et al. Frailty in elderly people. Lancet 2013;381:752-62.

4 Hoogendijk EO, Afilalo J, Ensrud KE, et al. Frailty: implications for clinical practice and public health. Lancet 2019;394:1365-75.

5 Puts MTE, Toubasi S, Andrew MK, et al. Interventions to prevent or reduce the level of frailty in community-dwelling older adults: a scoping review of the literature and international policies. Age Ageing 2017;46:383-92.

6 Ng TP, Feng L, Nyunt MSZ, et al. Nutritional, physical, cognitive, and combination interventions and frailty reversal among older adults: a randomized controlled trial. Am J Med 2015;128:1225-36.

7 Tarazona-Santabalbina FJ, Gómez-Cabrera MC, Pérez-Ros P, et al. A multicomponent exercise intervention that reverses frailty and improves cognition, emotion, and social networking in the community-dwelling frail elderly: a randomized clinical trial. J Am Med Dir Assoc 2016;17:426-33.

8 Apóstolo J, Cooke R, Bobrowicz-Campos E, et al. Effectiveness of interventions to prevent pre-frailty and frailty progression in older adults: a systematic review. JBI Database System Rev Implement Rep 2018;16:140-232. 
9 Daniels R, van Rossum E, de Witte L, et al. Interventions to prevent disability in frail community-dwelling elderly: a systematic review. BMC Health Serv Res 2008;8:278.

10 Cesari M, Vellas B, Hsu F-C, et al. A physical activity intervention to treat the frailty syndrome in older persons-results from the LIFE-P study. J Gerontol A Biol Sci Med Sci 2015;70:216-22.

11 Nagai K, Miyamato T, Okamae A, et al. Physical activity combined with resistance training reduces symptoms of frailty in older adults: a randomized controlled trial. Arch Gerontol Geriatr 2018;76:41-7.

12 Trombetti A, Hars M, Hsu F-C, et al. Effect of physical activity on frailty: secondary analysis of a randomized controlled trial. Ann Intern Med 2018;168:309-16.

13 Payette H, Boutier V, Coulombe C, et al. Benefits of nutritional supplementation in free-living, frail, undernourished elderly people: a prospective randomized community trial. J Am Diet Assoc 2002;102:1088-95.

14 Kim C-O, Lee K-R. Preventive effect of protein-energy supplementation on the functional decline of frail older adults with low socioeconomic status: a community-based randomized controlled study. J Gerontol A Biol Sci Med Sci 2013;68:309-16.

15 Dedeyne L, Deschodt M, Verschueren S, et al. Effects of multidomain interventions in (pre)frail elderly on frailty, functional, and cognitive status: a systematic review. Clin Interv Aging 2017;12:873-96.

16 Travers J, Romero-Ortuno R, Bailey J, et al. Delaying and reversing frailty: a systematic review of primary care interventions. $\mathrm{Br} J \mathrm{Gen}$ Pract 2019;69:e61-9.

17 Chan D-CD, Tsou H-H, Yang R-S, et al. A pilot randomized controlled trial to improve geriatric frailty. BMC Geriatr 2012;12:58.

$18 \mathrm{Ng} \mathrm{TP}$, Feng L, Nyunt MSZ, et al. Nutritional, physical, cognitive, and combination interventions and frailty reversal among older adults: a randomized controlled trial. Am J Med 2015;128-1225-36.

19 de Souto Barreto P, Rolland Y, Maltais M, et al. Associations of multidomain lifestyle intervention with frailty: secondary analysis of a randomized controlled trial. Am J Med 2018;131:1382.e7-1382.e13.

20 Oeseburg B, Wynia K, Middel B, et al. Effects of case management for frail older people or those with chronic illness: a systematic review. Nurs Res 2009;58:201-10.

21 You EC, Dunt D, Doyle C, et al. Effects of case management in community aged care on client and carer outcomes: a systematic review of randomized trials and comparative observational studies. BMC Health Serv Res 2012;12:395.

22 Hopman P, de Bruin SR, Forjaz MJ, et al. Effectiveness of comprehensive care programs for patients with multiple chronic conditions or frailty: a systematic literature review. Health Policy 2016;120:818-32.

23 Sadler E, Potterton V, Anderson R, et al. Service user, carer and provider perspectives on integrated care for older people with frailty, and factors perceived to facilitate and hinder implementation: a systematic review and narrative synthesis. PLoS One 2019;14:e0216488.

24 Looman WM, Huijsman R, Fabbricotti IN. The (cost-)effectiveness of preventive, integrated care for community-dwelling frail older people: A systematic review. Health Soc Care Community 2019;27:1-30.

25 Busse R, Stahl J. Integrated care experiences and outcomes in Germany, the Netherlands, and England. Health Aff 2014;33:1549-58.

26 Kojima G, Taniguchi Y, lliffe S, et al. Transitions between frailty states among community-dwelling older people: a systematic review and meta-analysis. Ageing Res Rev 2019;50:81-8.

27 Viktoria Stein K, Barbazza ES, Tello J, et al. Towards peoplecentred health services delivery: a framework for action for the world health organisation (who) European region. Int J Integr Care 2013;13:e058.

28 Woods NF, LaCroix AZ, Gray SL, et al. Frailty: emergence and consequences in women aged 65 and older in the women's health Initiative observational study. J Am Geriatr Soc 2005;53:1321-30.

29 Spoorenberg SLW, Wynia K, Fokkens AS, et al. Experiences of community-living older adults receiving integrated care based on the chronic care model: a qualitative study. PLoS One 2015;10:e0137803.

30 Wagner EH. Chronic disease management: what will it take to improve care for chronic illness? Eff Clin Pract 1998;1:2.

31 Wagner EH, Austin BT, Davis C, et al. Improving chronic illness care: translating evidence into action. Health Aff 2001;20:64-78.

32 Hendry A, Vanhecke E, Carriazo AM, et al. Integrated care models for managing and preventing frailty: a systematic review for the
European joint action on frailty prevention (advantage JA). Trans/ Med UniSa 2019;19:5-10.

33 Coleman K, Austin BT, Brach C, et al. Evidence on the chronic care model in the new millennium. Health Aff 2009;28:75-85.

34 World Health Organisation. Falls 2018, 2020. Available: https://www. who.int/news-room/fact-sheets/detail/falls [Accessed 7 Jun 2020].

35 Steventon AD, Friebel S, Gardner R. Emergency hospital admissions in England, 2020. Available: https://www.health.org.uk/publications/ emergency-hospital-admissions-in-england-which-may-beavoidable-and-how [Accessed 7 Jun 2020].

36 World Health Organisation. Whoqol: measuring quality of life 2020, 2020. Available: https://www.who.int/healthinfo/survey/whoqolqualityoflife/en/ [Accessed 7 Jun 2020].

37 Bowling A. The psychometric properties of the older people's quality of life questionnaire, compared with the CASP-19 and the WHOQOLOLD. Curr Gerontol Geriatr Res 2009;2009:298950.

38 Ware JE, Sherbourne CD. The MOS 36-item short-form health survey (SF-36). I. conceptual framework and item selection. Med Care 1992;30:473-83.

39 Skevington SM, Lotfy M, O'Connell KA, et al. The world Health organization's WHOQOL-BREF quality of life assessment: psychometric properties and results of the International field trial. A report from the WHOQOL group. Qual Life Res 2004;13:299-310.

40 Age UK. Care home 2020, 2020. Available: https://www.ageuk.org. uk/information-advice/care/arranging-care/care-homes/\# [Accessed 7 Jun 2020].

41 Gill TM, Gahbauer EA, Allore HG, et al. Transitions between frailty states among community-living older persons. Arch Intern Med 2006;166:418-23.

42 Fried LP, Ferrucci L, Darer J, et al. Untangling the concepts of disability, frailty, and comorbidity: implications for improved targeting and care. J Gerontol A Biol Sci Med Sci 2004;59:M255-63.

43 Jones DM, Song X, Rockwood K. Operationalizing a frailty index from a standardized comprehensive geriatric assessment. J Am Geriatr Soc 2004;52:1929-33.

44 Rothman MD, Leo-Summers L, Gill TM. Prognostic significance of potential frailty criteria. J Am Geriatr Soc 2008;56:2211-6.

45 Topinková E. Aging, disability and frailty. Ann Nutr Metab 2008;52 Suppl 1:6-11.

46 Morley JE, Vellas B, van Kan GA, et al. Frailty consensus: a call to action. J Am Med Dir Assoc 2013;14:392-7.

47 Cameron ID, Fairhall N, Langron C, et al. A multifactorial interdisciplinary intervention reduces frailty in older people: randomized trial. BMC Med 2013;11:65.

48 Theou O, Cann L, Blodgett J, et al. Modifications to the frailty phenotype criteria: systematic review of the current literature and investigation of 262 frailty phenotypes in the survey of health, ageing and retirement in Europe. Ageing Res Rev 2015;21:78-94.

49 de Vries NM, Staal JB, van der Wees PJ, et al. Patient-centred physical therapy is (cost-) effective in increasing physical activity and reducing frailty in older adults with mobility problems: a randomized controlled trial with 6 months follow-up. J Cachexia Sarcopenia Muscle 2016;7:422-35.

50 Bongue B, Buisson A, Dupre C, et al. Predictive performance of four frailty screening tools in community-dwelling elderly. BMC Geriatr 2017;17:262.

51 Van der Elst M, Schoenmakers B, Duppen D, et al. Interventions for frail community-dwelling older adults have no significant effect on adverse outcomes: a systematic review and meta-analysis. BMC Geriatr 2018;18:249.

52 Kojima G, Taniguchi Y, lliffe S, et al. Transitions between frailty states among community-dwelling older people: a systematic review and meta-analysis. Ageing Res Rev 2019;50:81-8.

53 Hébert R, Bravo G, Préville M. Reliability, validity and reference values of the Zarit burden interview for assessing informal caregivers of community-dwelling older persons with dementia. Can J Psychiatry 2000;19:494-507.

54 Higgins JP, Thomas J, Chandler J. Cochrane handbook for systematic reviews of interventions. John Wiley \& Sons, 2019.

55 The Cochrane Collaboration. EPOC resources for review authors, 2020. Available: https://epoc.cochrane.org/resources/epocresources-review-authors

56 L'Abbé KA, Detsky AS, O'Rourke K. Meta-analysis in clinical research. Ann Intern Med 1987;107:224-33.

57 Guyatt G, Oxman AD, Sultan S, et al. GRADE guidelines: 11. making an overall rating of confidence in effect estimates for a single outcome and for all outcomes. J Clin Epidemiol 2013;66:151-7. 